\title{
FORMATION OF A MATHEMATICAL MODEL AND ALGORITHM FOR IMPLEMENTATION OF CALCULATION MAIN PIPELINE - OF SHELL STRUCTURE
}

\author{
Abdusamat Abdusattarov ${ }^{1}$, Nodira Ruzieva ${ }^{1}$, Nizambay Sabirov ${ }^{2}$ \\ ${ }^{1}$ Tashkent State Transport University of the Republic of Uzbekistan, \\ Temiryo'lchilar st. 1, 100167, Tashkent, Uzbekistan \\ ${ }^{2}$ Tashkent Institute of Textile and Light Industry, \\ Shohdjahon st., 5, 100100, Tashkent, Uzbekistan
}

\begin{abstract}
Annotation. The paper considers methods of numerical calculation of elements of shell structures - main pipelines under various types of loading. Refined equations of motion for cylindrical shell structures are given. To solve boundary value problems of thin-walled structures, the Bubnov-Galerkin method, finite differences using the sweep method is used. As an example, the solution of the boundary value problem of a cylindrical shell under static loading by the finite difference method is given. And also the results of the study of the numerical convergence of the calculated values.

Key words: variational principle, mathematical model, algorithm, finite difference method, elasticity, deformation, shell, main pipeline.
\end{abstract}

\section{INTRODUCTION}

Shell theory is a very extensive branch of solid mechanics with a complex structure. Thin-walled shell structures (coatings and ceilings in construction, thermal power plants, gas and oil pipelines, pressure vessels, car bodies, tank boilers, tunnels) are distinguished by significant specificity of structural forms, manufacturing technology, operating conditions, physical and mechanical properties of the materials used [1-3].

Many issues related to the design of thin-walled structures and structures are led to the study of the stress-strain state of shell structures. Numerous results in the field of constructing a general theory of the shell were summarized in a number of monographs by famous scientists who played a large role in the development of this science and received world recognition: V.Z. Vlasov, A.L. Goldenveizer, A. Lyava, A. I. Lurie, VV Novozhilova, SP Timoshenko, SA Ambartsumyan, AS Volmira and others. When calculating shell structures, the methods of finite elements, finite differences and the method of boundary elements have become very widespread. Academician AASamarskiy made a significant contribution to the development of numerical modeling for solving applied problems [4]. He proposed the triad "model - algorithm - program", developed a methodology for a computational experiment and a technology for numerical modeling.

The article [2] analyzes the works of EI Grigolyuk and his contribution to the mechanics of a deformable solid, in particular, to the theory of shells and plates, to solving applied problems of strength of transport structures - aviation and rocket technology, nuclear energy, automobile structures.

In the work of VI Korolev [3], a method for calculating plates and shells under elastic and elastoplastic deformations is described. Reinforced plates, two-layer and multilayer shells, as well as shallow three-layer shells with elastic fillers are considered. In [5], finite element methods are considered, with the main attention being paid to the issues of the reliability of the results of calculations. Many computational examples illustrate the importance of theoretical inferences for practical calculations. The authors of [6] present modern continuum mechanics and mathematical foundations for studying the physical behavior of shells. Several modern finite element methods are presented for linear and nonlinear analyzes. The publications $[7,8]$ contain methods for solving nonlinear problems used in calculating the strength, stability and vibrations of structures. The calculations were performed taking into account physical and geometric nonlinearity using the variational method. A software product was created on the basis of the developed algorithm.

In [9], variational finite-difference methods for solving linear and nonlinear problems for thin-walled shells and plates made of homogeneous isotropic and orthotropic materials are considered. An analysis of the numerically stress-strain state of shells of complex geometry is given. The methods of successive approximations, the finite difference method and the numerical discretization algorithm are used. In the works of Acad. T. Burieva [10-11] considers the safety of structures of complex objects. Numerical methods for solving boundary value problems are being developed, issues of implementation on a computer, construction of an algorithmic system for calculating load-bearing structural elements within and beyond the limits of elasticity under variable loads. In work [12] it is noted that the constant improvement of strength methods for calculating thin-walled structures is associated with an increase in the degree of correspondence of the used mathematical models to the real conditions of their work. In [13], single and alternating bending of elastic-plastic three-layer rods and shells, asymmetric in thickness, was investigated.

The creation of software is one of the constituent parts of the algorithmic system for calculating thin-walled structures under cyclic loading, since the reliability of calculations and the reliability of design solutions for specific classes of problems directly depend on the adequacy of the chosen mathematical models, solution methods and boundary conditions.

\section{MATERIALS AND METHODS}


Following the theory of shells by VZ Vlasov [1], the displacement of an arbitrary point of the shell body, which lags behind the middle surface along the normal by a distance, can be represented in the form

$$
U_{\alpha}=\left(1+k_{1} \gamma\right) U-\frac{\gamma}{A} \cdot \frac{\partial W}{\partial \alpha}, U_{\beta}=\left(1+k_{2} \gamma\right) V-\frac{\gamma}{B} \cdot \frac{\partial W}{\partial \beta}, U_{\gamma}=W(\alpha, \beta) .
$$

Taking into account the expressions for the displacements (1), the Lamé coefficients and their ratios, we will write in the form of series in the variable $\gamma$ :

$$
\frac{1}{H_{2}}=\frac{1}{B}\left(1-k_{2} \gamma+k_{2}^{2} \gamma^{2}\right) ; \frac{H_{2}}{H_{1}}=\frac{B}{A}\left(1+k_{2} \gamma\right) ; \frac{H_{1}}{H_{2}}=\frac{A}{B}\left(1-k_{2} \gamma+k_{2}^{2} \gamma^{2}\right) ;
$$

to determine the deformations, the following refined formulas were obtained:

$$
\begin{gathered}
e_{\alpha \alpha}=\frac{1}{R} \frac{\partial U}{\partial \alpha}-\frac{\gamma}{R^{2}} \frac{\partial^{2} W}{\partial \alpha^{2}}, e_{\beta \beta}=\frac{\partial V}{R \partial \beta}-\left(\gamma-k_{2} \gamma^{2}\right) \frac{\partial^{2} W}{R^{2} \partial \beta^{2}}+\left(1-k_{2} \gamma+k_{2}^{2} \gamma^{2}\right) k_{2} W \\
e_{\alpha \beta}=\left(1-k_{2} \gamma+k_{2}^{2} \gamma^{2}\right) \frac{\partial U}{B \partial \beta}-\left(\gamma-k_{2} \gamma^{2}\right) \frac{\partial^{2} W}{A B \partial \alpha \partial \beta}+\left(1+k_{2} \gamma\right) \frac{\partial V}{A \partial \alpha}-\frac{\gamma}{A B} \frac{\partial^{2} W}{\partial \alpha \partial \beta} ;
\end{gathered}
$$

It is believed that the cylindrical shell deforms within the elastic range. Then the stress components are determined by the generalized Hooke's law

$$
\sigma_{\alpha}=(\lambda+2 \mu) \Delta-2 \mu\left(e_{\beta \beta}+e_{\gamma \gamma}\right) ; \sigma_{\beta}=(\lambda+2 \mu) \Delta-2 \mu\left(e_{\alpha \alpha}+e_{\gamma \gamma}\right) ; \tau_{\alpha \beta}=\mu e_{\alpha \beta} .
$$

To obtain the equation of motion for the elements of shell structures, the Hamilton - Ostrogradsky equation was used [14]

$$
\delta \int_{t}(\delta T-\delta \Pi+\delta \mathrm{A}) d t=0 .
$$

Variations in kinetic and potential energy and the work of external forces were determined by the following relationships:

$$
\begin{gathered}
\delta \Pi=\int_{V}\left(\sigma_{11} \delta \varepsilon_{11}^{Z}+\sigma_{12} \delta \varepsilon_{12}^{Z}+\sigma_{22} \delta \varepsilon_{22}^{Z}\right) d V \\
\delta A=\int_{V}\left(p_{1} \delta U_{1}+p_{2} \delta U_{2}+p_{3} \delta U_{3}\right) d V+\int_{S}\left(q_{1} \delta U_{1}+q_{2} \delta U_{2}+q_{3} \delta U_{3}\right) d S+ \\
+\left.\int_{S_{1}}\left(f_{1} \delta U_{1}+f_{2} \delta U_{2}+f_{3} \delta U_{3}\right) d S_{1}\right|_{\alpha_{1}}+\left.\int_{S_{2}}\left(\phi_{1} \delta U_{1}+\phi_{2} \delta U_{2}+\phi_{3} \delta U_{3}\right) d S_{2}\right|_{\alpha_{2}} \\
\delta \mathrm{T}=\int_{t} \int_{v}\left[\rho \frac{\partial u_{1}}{\partial t} \delta \frac{\partial u_{1}}{\partial t}+\rho \frac{\partial u_{2}}{\partial t} \delta \frac{\partial u_{2}}{\partial t}+\rho \frac{\partial u_{3}}{\partial t} \delta \frac{\partial u_{3}}{\partial t}\right] d v d t .
\end{gathered}
$$

To obtain the equation of motion for cylindrical shell structures, we used the Hamilton - Ostrogradsky variational principle (4) - (5). Taking into account the expressions for displacements (1), deformations (2), generalized Hooke's law (3), as well as performing integration by parts, introducing some notation from the variational equation, we obtained systems of differential equations of motion with boundary and initial conditions.

To solve boundary value problems, the Bubnov - Galerkin method was applied [14]:

$$
U=\sum_{n} U_{n}(\alpha, t) \cos \frac{n \pi \beta}{\beta_{1}}, V=\sum_{n} V_{n}(\alpha, t) \sin \frac{n \pi \beta}{\beta_{1}}, W=\sum_{n} W_{n}(\alpha, t) \cos \frac{n \pi \beta}{\beta_{1}} .
$$

After some transformations, a refined system of differential equations for cylindrical shells was obtained in the following form:

$$
\begin{gathered}
-\alpha_{1}^{(1)} \frac{\partial^{2} W_{n}}{\partial t^{2}}+\alpha_{2}^{(1)} \frac{\partial^{4} W_{n}}{\partial t^{2} \partial \alpha^{2}}-\alpha_{3}^{(1)} \frac{\partial^{2} V_{n}}{\partial t^{2}}-\alpha_{4}^{(1)} \frac{\partial^{4} W_{n}}{\partial \alpha^{4}}+\alpha_{5}^{(1)} \frac{\partial^{2} W_{n}}{\partial \alpha^{2}}-\alpha_{6}^{(1)} \frac{\partial U_{n}}{\partial \alpha}-\alpha_{7}^{(1)} W_{n}-\alpha_{8}^{(1)} V_{n}+Z_{n}=0 ; \\
-\alpha_{1}^{(2)} \frac{\partial^{2} U_{n}}{\partial t^{2}}+\alpha_{2}^{(2)} \frac{\partial^{2} U_{n}}{\partial \alpha^{2}}+\alpha_{4}^{(2)} \frac{\partial V_{n}}{\partial \alpha}+\alpha_{3}^{(2)} \frac{\partial W_{n}}{\partial \alpha}-\alpha_{5}^{(2)} U_{n}+X_{n}=0 ;
\end{gathered}
$$




$$
-\alpha_{2}^{3} \frac{\partial^{2} V_{n}}{\partial t^{2}}+\alpha_{1}^{(3)} \frac{\partial^{2} W_{n}}{\partial t^{2}}-\alpha_{4}^{(3)} \frac{\partial U_{n}}{\partial \alpha}+\alpha_{3}^{(3)} \frac{\partial^{2} V_{n}}{\partial \alpha^{2}}+\alpha_{5}^{(3)} W_{n}-\alpha_{6}^{(3)} V_{n}+Y_{n}=0
$$

border conditions:

$$
\begin{gathered}
{\left.\left[-(\lambda+2 \mu) \cdot h \frac{\partial U}{R \partial \alpha}-\lambda h \frac{\partial V}{R \partial \beta}-\lambda k_{2} \frac{h^{3}}{12} \frac{\partial^{2} W}{R^{2} \partial \beta^{2}}-\lambda \cdot\left(h+k_{2}^{2} \frac{h^{3}}{12}\right) k_{2} W+N\left(\phi_{1}\right)\right] \delta U\right|_{\alpha}=0} \\
{\left.\left[-\mu\left(h+k_{2}^{2} \frac{h^{3}}{12}\right) \frac{\partial U}{R \partial \beta}-\mu h \frac{\partial V}{R \partial \alpha}-\mu k_{2} \frac{h^{3}}{12} \frac{\partial^{2} W}{R^{2} \partial \alpha \partial \beta}+N\left(\phi_{2}\right)\right] \delta V\right|_{\alpha}=0} \\
{\left[(\lambda+2 \mu) \frac{h^{3}}{12} \frac{\partial^{3} W}{R^{3} \partial \alpha^{3}}+\lambda \frac{h^{3}}{12} \frac{\partial^{3} W}{R^{3} \partial \alpha \partial \beta^{2}}+\lambda k_{2}^{2} \frac{h^{3}}{12} \frac{\partial W}{R \partial \alpha}+2 \mu k_{2} \frac{h^{3}}{12} \frac{\partial^{2} U}{R^{2} \partial \beta^{2}}-\right.} \\
\left.-\mu k_{2} \frac{h^{3}}{12} \frac{\partial^{2} V}{R^{2} \partial \alpha \partial \beta}+4 \mu \frac{h^{3}}{12} \frac{\partial^{3} W}{R^{3} \partial \alpha \partial \beta^{2}}+Q\left(\phi_{3}\right)+\frac{\partial M\left(\phi_{2}\right)}{R \partial \beta}\right]\left.\delta W\right|_{\alpha}=0 \\
{\left.\left[-(\lambda+2 \mu) \frac{h^{3}}{12} \frac{\partial^{2} W}{R^{2} \partial \alpha^{2}}-\lambda \frac{h^{3}}{12} \frac{\partial^{2} W}{R^{2} \partial \beta^{2}}-\lambda k_{2}^{2} \frac{h^{3}}{12} W-M\left(\phi_{1}\right)\right] \delta \frac{\partial W}{R \partial \alpha}\right|_{\alpha}=0 .}
\end{gathered}
$$

initial conditions:

$$
\begin{aligned}
\left.\rho h \frac{\partial U}{\partial t} \delta U\right|_{t}=0 ;[ & {\left.\left[\left(h+k_{2}^{2} \frac{h^{3}}{12}\right) \frac{\partial V}{\partial t}-\rho k_{2} \frac{h^{3}}{12} \frac{\partial^{2} W}{\partial t R \partial \beta}\right] \delta V\right|_{t}=0 ; } \\
& {\left.\left[\rho h \frac{\partial W}{\partial t}-\rho \frac{h^{3}}{12} \frac{\partial^{3} W}{\partial t R^{2} \partial \alpha^{2}}+\rho k_{2} \frac{h^{3}}{12} \frac{\partial^{2} V}{\partial t R \partial \beta}-\rho \frac{h^{3}}{12} \frac{\partial^{3} W}{\partial t R^{2} \partial \beta^{2}}\right] \delta W\right|_{t}=0 . }
\end{aligned}
$$

The system of differential equations (9) can be written in vector form

$$
A_{1} \ddot{U}_{n}+A_{2} \ddot{U}_{n}^{I I}+A_{3} U_{n}^{I V}+A_{4} U_{n}^{I I}+A_{5} U_{n}^{I}+A_{6} U_{n}+F_{n}=0
$$

where $U_{k}=\left(W_{k}, U_{k}, V_{k}\right)^{T}, F_{k}=\left(Z_{k}, X_{k}, Y_{k}\right)^{T} ; A_{i}-$ matrix of the third order.

To solve boundary value problems (12), (10), and (11), the method of finite differences of the second order of accuracy is used [4]. Based on the use of central difference formulas, the following system of algebraic equations was obtained:

$$
\begin{gathered}
B_{n} U_{n, i-1}^{k+1}+C_{n} U_{n, i}^{k+1}+B_{n} U_{n, i+1}^{k+1}+\bar{A}_{n} U_{n, i+1}^{k+1}+\bar{B}_{n} U_{n, i-1}^{k}+\bar{C}_{n} U_{n, i}^{k}+\bar{D}_{n} U_{n, i+1}^{k}+\bar{A}_{n} U_{n, i+2}^{k}+B_{n} U_{n, i-1}^{k-1}+ \\
+C_{n} U_{n, i}^{k-1}+B_{n} U_{n, i+1}^{k-1}+\tau^{2} F_{n, i}^{k}=0 .
\end{gathered}
$$

After approximation, the initial condition (11) takes the following form:

$$
\left[\bar{M}_{1} U_{n, i-1}^{k+1}+\bar{M}_{2} U_{n, i}^{k+1}+\bar{M}_{3} U_{n, i+1}^{k+1}-\bar{M}_{1} U_{n, i+1}^{k+1}-\bar{M}_{1} U_{n, i-1}^{k-1}-\bar{M}_{2} U_{n, i}^{k-1}-\bar{M}_{3} U_{n, i+1}^{k-1}\right] \cdot t_{0} h \delta U_{n, i+1}^{k-1}=0 .
$$

The difference boundary value problem is solved by the sweep method. It is assumed that the displacements and their velocities at the initial moment of time are given. It is believed that the cylindrical shell is clamped at $\alpha=0$ and $\alpha=1$. In vector form, the boundary conditions are expressed as follows way:

$$
U_{n, 0}^{j}=0 ; A^{\prime} U_{n,-1}^{j}=A^{\prime} U_{n, 1}^{j} ; U_{n, N}^{j}=0 ; A^{\prime} U_{n, N+1}^{j}=A^{\prime} U_{n, N-1}^{j} .
$$

We rewrite the system of difference equations (13) taking into account the boundary conditions (15) at $i=1,2, \ldots, N-1$; as a result, the system takes the form

$$
B_{n} U_{n, i-1}^{k+1}+C_{n} U_{n, i}^{k+1}+B_{n} U_{n, i+1}^{k+1}=b_{n, j},
$$


где

$b_{n, i}=\tau^{2} F_{n, i}^{k}-\left(\bar{A}_{n} U_{n, i-2}^{k}+\bar{B}_{n} U_{n, i-1}^{k}+\bar{C}_{n} U_{n, i}^{k}+\bar{D}_{n} U_{n, i+1}^{k}+\bar{A}_{n} U_{n, i+2}^{k}+B_{n} U_{n, i-1}^{k-1}+C_{n} U_{n, i}^{k-1}+B_{n} U_{n, i+1}^{k-1}\right)$.

From equation (16), we can derive a solution for the ith equation

$$
\begin{gathered}
U_{n, i}^{K+1}=f_{i}-H_{i} U_{n, i+1}^{K+i}, \\
\text { где } f_{i}=\left(C_{n}-B_{n} H_{i-1}\right)^{-1}\left(b_{n, i}-B_{n} f_{i+1}\right), H_{i}=\left(C_{n}-B_{n} H_{i-1}\right)^{-1} B_{n} .
\end{gathered}
$$

During the reverse sweep, the remaining values of the displacement vector are determined $U_{n, i}^{k}$.

\section{CALCULATION RESULTS AND DISCUSSION}

On the basis of the given algorithm, a complex program and computer implementation of solving problems of a cylindrical shell $[15,16]$ have been developed.

The created complex of programs $(\mathrm{C} \#)$ consists of several classes. Class is a keyword in Object Oriented Programming (OOP). In OOP, a class is the main element within which the design of programs is carried out. A class contains data and code that manages this data. A class constructor is a special method that is called when a new object is created and is used to initialize the class fields with values, as well as for initial calculations, if necessary.

The complex of programs works in an interactive mode. The created interface assumes both the output of the calculation results in the form of tables and graphs, and their recording in individual files for their further consideration and analysis [17-20]. As an example, the solution of the boundary value problem of a cylindrical shell under static loading by the finite difference method is given, as well as the study of the numerical convergence of the calculated values. Geometric and mechanical characteristics of the cylinder: $\mathrm{h}=0.01 \mathrm{~cm} ; \mu=0.3 ; \mathrm{R}=150 \mathrm{~cm} ; L=1120 \mathrm{~cm} ; \beta=2 \pi ; \mathrm{q}=1$; $E=2 \cdot 10^{5} \mathrm{MPa}$. The obtained dimensionless results are presented in the form of tables and graphs (Fig. 1).

\begin{tabular}{|c|c|c|c|c|c|c|}
\hline$\alpha$ & $W$ & $U$ & $\mathrm{M}_{1}$ & $\mathrm{M}_{2}$ & $\mathrm{~N}_{1}$ & $\mathrm{~N}_{2}$ \\
\hline 0 & 0,0 & 0,0 & $-9,71608$ & $-4,17511$ & $-2,16607$ & $-0,99421$ \\
\hline 0.1 & $-0,05403$ & $-0,87381$ & $-3,56012$ & $-1,80509$ & $-1,07511$ & $-2,58409$ \\
\hline 0.2 & $-0,16721$ & 0,06788 & 0,07746 & $-0,30733$ & $-0,35142$ & $-6,17013$ \\
\hline 0.3 & $-0,26960$ & 2,62508 & 2,15907 & 0,63612 & $-0,63503$ & $-10,11210$ \\
\hline 0.4 & $-0,34052$ & 5,70209 & 3,28106 & 1,24514 & $-1,78410$ & $-13,10121$ \\
\hline 0.5 & $-0,36473$ & 8,34110 & 3,53810 & 1,58013 & $-3,17805$ & $-14,11012$ \\
\hline
\end{tabular}

The nature of the change in the calculated values along the length $(\alpha)$ of the cylindrical shell
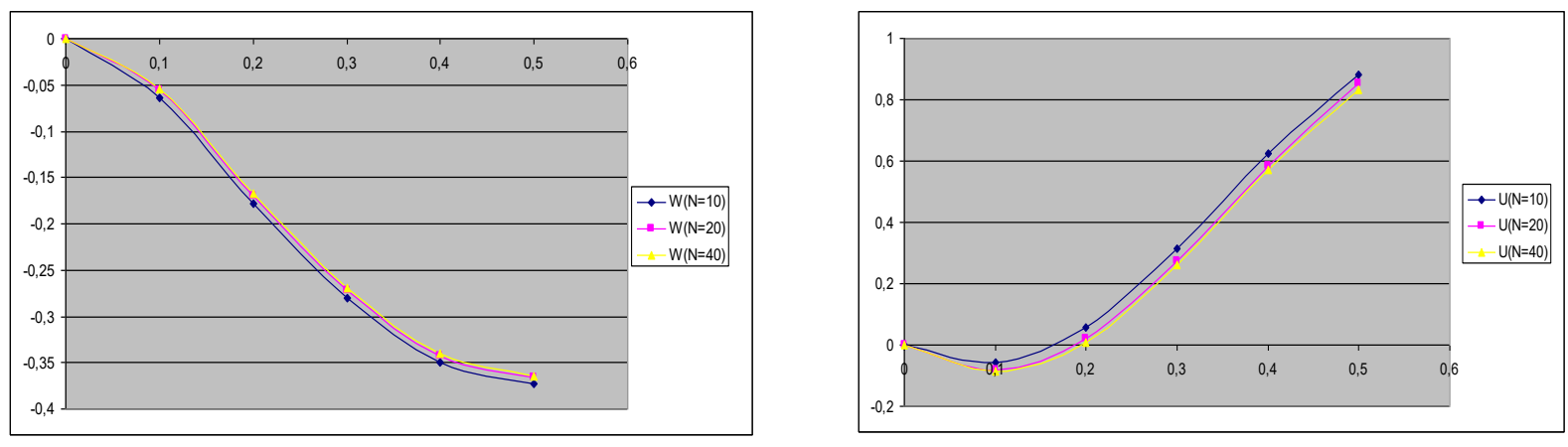

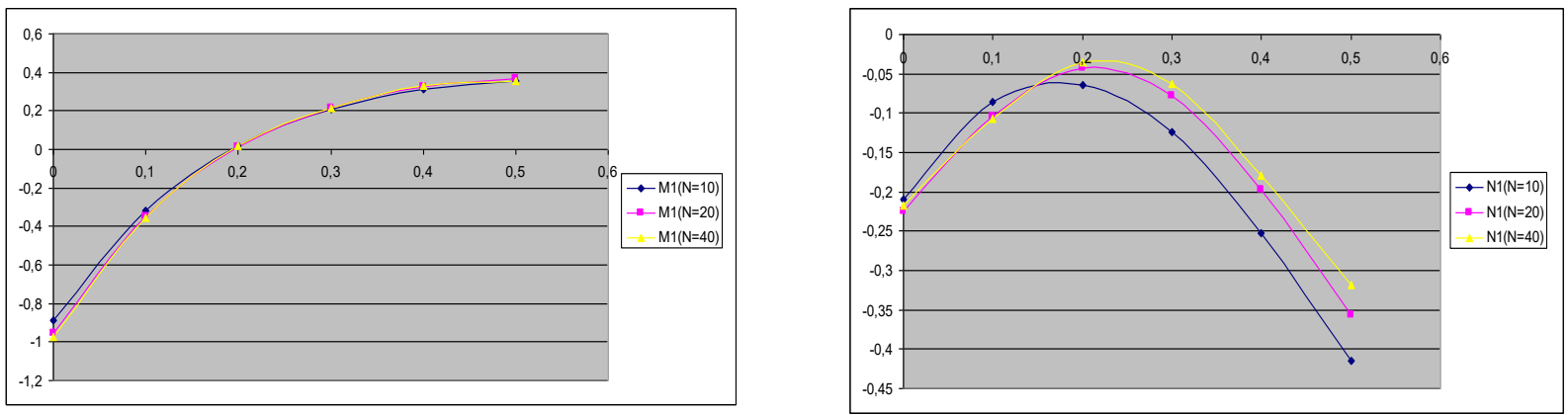

Fig. 1. The nature of the change in the calculated values and the convergence of the parameters for different values of the grid spacing $h$

For a comparative analysis, the calculation of the stress-strain state of a composite shell structure - a main pipeline using the ANSYS complex. 


\section{CONCLUSIONS}

Equations of motion of cylindrical shell structures - main pipelines are obtained taking into account the refined geometric materials according to V.Z. Vlasov. To solve systems of differential equations with boundary and initial conditions, discrete models have been developed based on the combined Bubnov - Galerkin method and finite differences. The developed program and the interface of the program for outputting the calculation results in the form of a table and graphs. As examples of displacements and forces for cylindrical shells fixed on both sides.

The developed algorithms and software have been tested and implemented at the facilities of the Tashkent metroproekt, Uzogirsanoat-loyiha and Foundry-Mechanical Plant during the design and survey work.

\section{REFERENCES}

1. 1. Vlasov V.Z. General theory of shells and its applications in technology -M .: Gostekhizdat, 1949. p.761.

2. 2. Grigolyuk EI, Lopanitsyn EA Final deflections, stability and supercritical behavior of thin shallow shells $-\mathrm{M}$.: MGTU "MAMI", 2004. p.62.

3. 3. Korolev VI Elastoplastic deformations of shells. -M .: Mashinostroenie, 1971.p.303.

4. 4. Samarskiy A.A., Mikhailov A.P. Mathematical Modeling: Ideas. Methods. Examples. -M .: Nauka, 2001.p.316

5. Babuska I. and Strouboulis T. The Finite Element Method and Its Reliability. Oxford: Oxford University Press, 2001. -802 pp.

6. Bathe K. J. and Chapelle D. Finite Element Methods for Shells. Berlin: Springer Verlag., 2002. - 400 pp.

7. Karpov V.V., Maslennikov A.M. Methods for Solving Non-Linear Tasks for Calculating Construction Structures // World Applied Sciences Journal, 23(Problems of Architecture and Construction). 2013. Pp: 178-183. http://idosi.org/wasj/wasj23/28pac/2913/35.pdf DOI: 10.5829/idosi. wasj. 2013.23 .pac.90035.

8. Karpov, V. and A. Semenov, 2014. Strength and Stability of Orthotropic Shells. World Applied Sciences Journal, 30 (5), Pp: 617-623. http://www.idosi.org/wasj/wasj30(5)14/14.pdf DOI: 10.5829/ idosi.wasj. 2014.30.05. 14064.

9. Maksimyuk V.A., Storozhuk E.A., Chernyshenko I.S. Variational finite-difference methods in linear and nonlinear problems of the deformation of metallic and composite shells (review) // International Applied Mechanics. 2012. Volume 48, Issue 6. Pp. 613-687. DOI: 10.1007/s 10778-012-0544-8.

10. Buriev T. Algorithmization of calculation of load-bearing elements of thin-walled structures. T .: Ed. "Fan", 1986, - p.244.

11. Buriev T., Abdusattarov A. Development of algorithms for calculating load-bearing elements of structures under variable loading taking into account hardening-softening and accumulation of damage // Reports of the Academy of Sciences of the Republic of Uzbekistan, 2000. - №2. -S. 13-17.

12. Grigorenko Ya.M., Vlaikov G.G., Grigorenko A.Ya. Numerical-analytical solution of problems of shell mechanics based on various models // -Kiev: Akademperiodika, 2006. p.472 .

13. Starovoitov E.I. Viscoelastoplastic layered plates and shells. - Gomel: BelGUT, 2002. p.344.

14. Kabulov V.K. Algorithmization in Continuum Mechanics. - Tashkent: Fan, 1979 p. 304 .

15. Myachenkov V.I., Grigoriev I.V. Calculation of composite shell structures on a computer. $-\mathrm{M}$.: Mechanical engineering, 1981. p.216.

16. Abdusattarov A., Yuldashev T., Matkarimov A.Kh., Isomiddinov A.I. Modeling the processes of deformation and damage of thin-walled structures. Tashkent, "Uzbekistan", 2012, p.153.

17. Abdusattarov A., Abdukadirov F.E., Sabirov N.Kh. Investigation of SSS and Natural Vibrations of Shell Structures Taking into Account the Inelastic Properties of Materials // Uzbek. journal "Problems of Mechanics", 2020, №1 / 2, p.24-30.

18. Abdusattarov A., Sabirov N.Kh. Investigation of the stress-strain state of a cylindrical shell - a tank boiler according to the semi-momentless theory // Uzbek. magazine "Problems of Mechanics", 2019, №2. - P.3-8.

19. Abdusattarov A., Isomiddinov A.I., Ruzieva N.B. Equation of motion of underground main pipelines under spatially variable elastic-plastic loading. // Modern architecture, strength of buildings and structures. Reliability and seismic safety problems. Materials of the Republican Scientific and Practical Conference. NamISI, 2021,135-137.

20. Abdusattarov A., Abdukadirov F.E., Sabirov N.X. - "Numerical calculation of elements of thin-walled structures under alternating loading taking into account damage" in IOP Conf. Series: Journal of Physics: Conf. Series 1479 (2020) 012143 